\title{
A Protocol Analysis of Brand Choice Strategies Involving Recommendations
}

\author{
DENNIS L. ROSEN \\ RICHARD W. OLSHAVSKY*
}

\begin{abstract}
Subjects completed a purchase decision under a computer-controlled laboratory simulation that enabled acquisition of both attribute-value and recommendation information from five sources. Analysis of subjects' acquisitions and recorded statements during the decision process offered support for the existence of unique decision strategies for situations involving availability of both kinds of information. Based on the manner in which recommendation information was used, these strategies were subdivided into (1) a recommendation-forms-evoked-set (RFES) for consideration, and (2) a recommendation-forms-standard (RFS) for comparison to other brand alternatives. Differences in strategy use between two product categories were explored.
\end{abstract}

$\mathrm{M}$ uch laboratory research on consumer decision making and information processing has involved settings where only attribute-value type information is presented, typically from a single source (e.g., Bettman and Jacoby 1976; Painton and Gentry 1985; Russo and Rosen 1975). However, in the real world, the consumer might request brand recommendations from one or more sources to help in the decision process. This study investigated the specific processing strategies that are used when both attribute-value and recommendation information are made available.

\section{RECOMMENDATION USE IN DECISION STRATEGIES}

The study of recommendation use has been typically subsumed under the topic of interpersonal influence. However, studies of "opinion leaders" or "reference groups" are generally concerned with the broad range of influence involved (Bearden and Etzel 1982; Park and Lessig 1977). Thus, the potential importance of a recommendation as a unique kind of information has been lost.

Its importance is emphasized by Olshavsky and Granbois (1979), who state that the consumer may obtain a recommendation from a personal or nonpersonal source with the intention of purchasing the recommended brand without acquiring attribute-value infor-

${ }^{*}$ Dennis L. Rosen is Associate Professor of Business at the School of Business, University of Kansas, Lawrence, KS 66045. Richard W. Olshavsky is Professor of Marketing at the Graduate School of Business, Indiana University, Bloomington, IN 47405. The authors are grateful to anonymous reviewers for their comments and to Paul Overhauser for programming the computer simulation. mation. This "subcontracted" decision making, as it will be referred to here, may replace traditional "ownbased" decision making when the consumer feels constraints on his/her time or recognizes the referrer as a particularly knowledgeable source.

In a second proposed recommendation-use strategy called "hybrid" (Olshavsky and Granbois 1979), the consumer, while making the final brand decision, allows one or more referrers to become involved in the decision through their recommendations. For example, the consumer may limit subsequent information search to only those brands recommended by the referrer(s) (Olshavsky and Rosen 1985). We will refer to this as the "recommendation-forms-evoked-set" (RFES) strategy. In another hybrid strategy, the consumer might allow the recommended brand to become a standard for comparison to other brands in his/her evoked set. S/he would then try to find another brand that is just as good as the standard but is superior on an attribute of interest such as price. We will refer to this as the "recommendation-forms-standard" (RFS) strategy. Solomon's (1986) recent discussion of the surrogate consumer indicates the need for empirical study of these potential strategies.

Our previous research (Olshavsky and Rosen 1985; Rosen and Olshavsky 1987) has indicated some support for the existence of a general hybrid decision making process and limited support for subcontracted processing. The results were consistent with the view that these strategies can be used as a method of limiting information acquisition activity. In the current study, a protocol analysis was added to aid in clarifying choice strategies used in the presence of both attribute-value and recommendation information. We hypothesized that both subcontracted and hybrid strategies, including 
RFES and RFS, would be in evidence and that less information acquisition would occur when these strategies were used. Differences in strategy use between two product categories were explored.

\section{METHOD}

Thirty-four seniors from business classes chose brands from the product category to which they were randomly assigned, either frozen pizza or stereo receivers. The product categories were chosen because of subject familiarity and differences in product characteristics. Information was presented to subjects via an interactive computer display program. The process was similar to that used in our previous studies, except that in this study, an assistant blind to the hypotheses entered the necessary codes for the subject's information requests. The subject then observed the information on the CRT screen. Information sources available were Advertising, Friend (described as one who is trusted and knowledgeable), Consumer Reports, In-Store Inspection (of product and package for pizza; product and brochure for stereo), and Salesperson/Clerk.

Time delays before a requested source of information became accessible were incorporated and ranged from 60 seconds (Advertising) to 230 seconds (Consumer Reports). While the delays were based on research concerning the effort involved in obtaining information in the real world (Duncan and Olshavsky 1982), we realized that they had very limited generalizability to the real world. Despite this fact, we felt that it was important to incorporate some form of information cost as has been suggested by Hoyer and Jacoby (1983). The delays were listed along with the source options on the first screen of the computer display.

Following source access, information options allowed the subjects to request separate lists of all brands or attributes with which the source was familiar. The numbers of brands and attributes varied by source (held constant across products) reflecting the real world. In total, 17 fictitious brand names were involved for each product. Attributes totaled 18 for stereo and 24 for pizza and reflected real-world information. Subjects could request, through the experimenter, specific attribute information for specific brands. Pictures of the products were not provided.

Also available for request by subjects was a statement of which brand the source recommended. Friend, Consumer Reports, and Salesperson/Clerk each recommended a different brand. A recommendation request from Advertising or In-Store Inspection produced the response, "whichever brand is being advertised (or whichever brand you are looking at) at the time." While not useful to the subjects, this response reflected the form recommendations take for these sources in the marketplace (e.g., an ad recommends the brand it is advertising).
Subjects were free to acquire as much or as little information as desired from a source and could select additional sources with an associated time delay invoked with source change. A pad and a pencil were available for recording information if desired. Brand choice for a practice product enabled development of subject familiarity with the computer-based information system prior to actual data collection. (See Olshavsky and Rosen (1985) and Rosen and Olshavsky (1987) for additional details on the computer-simulation methodology.)

Subjects were asked to "think aloud" as they went through the entire process; their statements were tape recorded. At the end of the session, a measure of product knowledge on a five-point scale was obtained.

\section{ANALYSIS AND RESULTS}

We reviewed tape transcriptions independently for classification of decision strategies according to the following predetermined scheme:

1. Own-based decision making-recommendation information was not considered.

2. Subcontracted decision making-a single recommendation was acquired and that brand purchased with no other information acquisition.

3. RFES - the subject only acquired attribute-value information about one or more brands that had been previously recommended and "purchased" one of the recommended brands.

4. RFS-recommendation was used to establish a brand standard with attempts then made to find a comparable "better buy."

Recommendation use was defined by acquisition of a brand specific recommendation. While a positive response on a general attribute, like "taste" or "sound quality," might have been construed by subjects as a recommendation, it was not possible to confirm such behavior in the current study. Since the product condition was evident when coding the protocols, great care was taken to follow the established classification guidelines. Four ambiguous protocols were dropped from analysis.

\section{Findings-The Hypotheses}

Tables 1 and 2 summarize information accessed and processing strategy by product type for each subject. Twenty of the classified subjects (66.7 percent) demonstrated own-based decision making. Only six of these 20 subjects acquired any recommendation information.

None of the subjects employed a strict subcontracting rule, though one subject acquired only price information for the recommended brand before its purchase. 
TABLE 1

INFORMATION ACCESS OF OWN-BASED STRATEGY SUBJECTS

\begin{tabular}{|c|c|c|c|c|c|c|c|c|c|c|c|c|c|c|c|}
\hline \multirow[b]{3}{*}{ Subject } & \multirow[b]{3}{*}{ Product } & \multicolumn{10}{|c|}{ Sources accessed ${ }^{\mathrm{a}}$} & \multirow[b]{3}{*}{ Processing ${ }^{\circ}$} & \multirow{2}{*}{\multicolumn{3}{|c|}{ Amount accessed }} \\
\hline & & \multicolumn{5}{|c|}{ Recommendations } & \multicolumn{5}{|c|}{ Information } & & & & \\
\hline & & $A D$ & $\mathrm{CR}$ & FR & is & SL & $A D$ & CR & FR & is & SL & & Brands & Attributes & Information \\
\hline 1 & Pizza & & & & $x$ & & & & $x$ & $x$ & & A & 7 & 5 & 15 \\
\hline 2 & Pizza & & $x$ & & & & & & $x$ & & $x$ & A & 7 & 2 & 13 \\
\hline 3 & Pizza & & & & & & $x$ & & & $x$ & & A & 6 & 7 & 13 \\
\hline 4 & Pizza & & & & & & $x$ & & $x$ & & & A & 10 & 9 & 27 \\
\hline $5^{c}$ & Pizza & & & $x$ & & & $x$ & & $x$ & & & A & 7 & 3 & 8 \\
\hline 6 & Pizza & $x$ & & $x$ & & & $x$ & & $x$ & & & A & 5 & 9 & 19 \\
\hline 7 & Pizza & & & & & & $x$ & $x$ & $x$ & $x$ & $x$ & A & 17 & 21 & 66 \\
\hline 8 & Pizza & & & & & & $x$ & & & $x$ & & A & 6 & 6 & 18 \\
\hline 9 & Pizza & & & & & & & & & $x$ & & B & 2 & 3 & 5 \\
\hline 10 & Pizza & & & & & & & & $x$ & & & A & 3 & 5 & 11 \\
\hline 11 & Pizza & & & & & & $x$ & & $\hat{x}$ & $x$ & & A & 4 & 6 & 10 \\
\hline 12 & Pizza & & & & & & & $x$ & & & & $A / B$ & 3 & 5 & 7 \\
\hline 13 & Pizza & & & & & & & & $x$ & $x$ & & A & 9 & 8 & 41 \\
\hline 14 & Stereo & & & & & & $x$ & & $x$ & & $x$ & A & 13 & 17 & 77 \\
\hline 15 & Stereo & & & & & & $x$ & & & $x$ & $x$ & A & 5 & 14 & 28 \\
\hline 16 & Stereo & & & & & & $x$ & & $x$ & $x$ & $x$ & $A / B$ & 7 & 7 & 14 \\
\hline 17 & Stereo & & & & & & $x$ & $x$ & $x$ & & & A & 6 & 9 & 44 \\
\hline 18 & Stereo & & & & & & & $x$ & $x$ & & & B & 7 & 17 & 70 \\
\hline 19 & Stereo & & $x$ & & & & & $x$ & $x$ & & & B & 2 & 8 & 12 \\
\hline \multirow[t]{3}{*}{20} & Stereo & $\times$ & & $x$ & $x$ & $x$ & $x$ & & $x$ & $x$ & $x$ & $A$ & 10 & 21 & 71 \\
\hline & & \multicolumn{10}{|c|}{ Number of subjects accessing } & & \multicolumn{3}{|c|}{ Mean access } \\
\hline & & 2 & 2 & 3 & 2 & 1 & 12 & 5 & 15 & 10 & 6 & & 6.80 & 9.10 & 28.45 \\
\hline
\end{tabular}

- $A D=$ Advertising; $C R=$ Consumer Reports; FR = Friend; IS = In-Store Inspection; SL = Salesperson/Clerk. Sources for which only brand or attribute lists were assessed by a subject are not included.

- $A=$ by attribute; $B$ = by brand.

' First access, not including brand or attribute listings, was a recommendation from Consumer Reports, Friend, or Salesperson/Clerk.

Ten of the classified subjects showed evidence of hybrid decision making. Hybrid strategies employed could be classified into two separate groups as had been hypothesized. Seven subjects demonstrated use of RFES hybrid processing and, in most cases, started with acquisition of a recommendation that formed the basis for further exploration. Two of these seven subjects demonstrated a shift to RFES following an initial short period of attribute-value acquisition. Three subjects demonstrated use of RFS hybrid processing. No other distinct processing strategies were observed. Of the 15 subjects in the Stereo condition, eight (53.3 percent) used a hybrid strategy while only two (13.3 percent) of the 15 subjects in the Pizza condition used a hybrid strategy. This difference was significant $(p=0.05)$ using a two-tailed Fisher Exact Probability Test.

We had hypothesized less information acquisition for subjects using the hybrid strategies. Through recommendation acquisition, RFES subjects were quickly able to limit any further consideration to a small subset of the original brand alternatives, thus acquiring, on average, less information than own-based subjects. A reduction in acquisition was not as obvious for the three RFS subjects who needed to acquire considerable in- formation in their efforts to find a better alternative than the standard.

This is reflected in mean comparisons for access of brands, attributes, and unique information as found on Tables 1 and 2. Means across all hybrid subjects were lower than those for the own-based subjects, but onetailed $t$-tests were nonsignificant for brand access ( $t$ $=-1.20, \mathrm{df}=15$, n.s.) and only marginally significant $(p<0.10)$ for attribute access $(t=-1.41, \mathrm{df}=22)$ and total information (brand/attribute combinations) access $(t=-1.48, \mathrm{df}=24)$. However, with RFS subjects removed, the difference between RFES and own-based subjects was as expected and highly significant ( $t$ $=-5.44, \mathrm{df}=23, p<0.001$ for brand access, $t=-2.57$, $\mathrm{df}=21, \mathrm{p}<0.01$ for attribute access, $t=-3.73$, df $=23, p<0.001$ for total information access; separate variance estimates were used in all tests).

Most of the hybrid subjects ( 8 of 10) acquired a recommendation prior to any acquisition of attribute-value information while only one of the 20 own-based subjects did so. This is consistent with a decision simplification view for RFES subjects and a comparison-to-standard view for RFS subjects since early acquisition of the recommendation in both cases would be most efficient. 
TABLE 2

INFORMATION ACCESS OF HYBRID STRATEGY SUBJECTS

\begin{tabular}{|c|c|c|c|c|c|c|c|c|c|c|c|c|c|c|c|}
\hline \multirow[b]{3}{*}{ Subject } & \multirow[b]{3}{*}{ Product } & \multicolumn{10}{|c|}{ Sources accessed ${ }^{\mathrm{a}}$} & \multirow[b]{3}{*}{ Processing $^{b}$} & \multirow{2}{*}{\multicolumn{3}{|c|}{ Amount accessed }} \\
\hline & & \multicolumn{5}{|c|}{ Recommendations } & \multicolumn{5}{|c|}{ Information } & & & & \\
\hline & & $A D$ & CR & $\mathrm{FR}$ & IS & SL & $A D$ & CR & FR & IS & SL & & Brands & Attributes & Information \\
\hline \multicolumn{16}{|l|}{ RFES } \\
\hline & Stereo & & & $x$ & & & & & $x$ & $x$ & $x$ & $\mathrm{~B}$ & 1 & 5 & 5 \\
\hline $2^{c}$ & Stereo & & $x$ & $x$ & & & & $x$ & $x$ & $x$ & & B & 2 & 9 & 14 \\
\hline $3^{c}$ & Stereo & & & $x$ & & $x$ & $x$ & $x$ & & & $x$ & $\mathrm{~B}$ & 2 & 8 & 15 \\
\hline 4 & Pizza & & & $x$ & & & & & $x$ & & & $\mathrm{~A} / \mathrm{B}$ & 3 & 4 & 6 \\
\hline 5 & Stereo & & $x$ & $x$ & & & & $x$ & $x$ & & & A & 3 & 2 & 3 \\
\hline $6^{c}$ & Pizza & & & $x$ & & & & & $x$ & & & -0 & 1 & 1 & 1 \\
\hline $7^{c}$ & Stereo & & & $x$ & & $x$ & & & $x$ & $x$ & $x$ & B & 2 & 4 & 4 \\
\hline \multicolumn{16}{|l|}{ RFS } \\
\hline $8^{c}$ & Stereo & & & $x$ & & $x$ & & & $x$ & $x$ & $x$ & $B$ & 10 & 18 & 46 \\
\hline $9^{c}$ & Stereo & & & $x$ & & & & $x$ & $x$ & $x$ & & A & 12 & 6 & 31 \\
\hline \multirow[t]{3}{*}{$10^{\circ}$} & Stereo & & & $x$ & & & & $x$ & $x$ & & & A & 12 & 6 & 45 \\
\hline & & \multicolumn{10}{|c|}{ Number of subjects accessing } & & \multicolumn{3}{|c|}{ Mean access } \\
\hline & & 0 & 2 & 10 & 0 & 3 & 1 & 5 & 9 & 5 & 4 & $\begin{array}{l}\text { Hybrid } \\
\text { RFES } \\
\text { RFS }\end{array}$ & $\begin{array}{r}4.80 \\
2.00 \\
11.33\end{array}$ & $\begin{array}{r}6.30 \\
4.71 \\
10.00\end{array}$ & $\begin{array}{r}17.00 \\
6.86 \\
40.67\end{array}$ \\
\hline
\end{tabular}

- $A D=$ Advertising; $C R=$ Consumer Reports; $F R=$ Friend; IS = In-Store Inspection; SL = Salesperson/Clerk. Sources for which only brand or attribute lists were accessed by a subject are not included.

${ }^{\circ} \mathrm{A}=$ by attribute; $\mathrm{B}=$ by brand

' First access, not including brand or attribute listings, was a recommendation from Consumer Reports, Friend, or Salesperson/Clerk.

- Undefined due to access of a single unit of information about a single brand within the sources involved.

\section{Additional Findings}

Effect of Product Knowledge. A $t$-test for the product knowledge rating (five-point, high (1) to low (5) scale) was conducted for 29 subjects for which these data were available. It indicated significantly greater knowledge in the product area for those using the own-based strategy than for those using a hybrid strategy (means of 3.15 and 3.89, respectively; $t=-1.99$, df $=23, p$ $=0.06$ ). However, this difference in knowledge ratings probably reflects the greater use of the own-based strategy by those in the Pizza condition and greater use of hybrid strategies by those in the Stereo condition. When the own-based versus hybrid comparison on knowledge was made for only those subjects in the Stereo condition, the means were identical (4.00).

Brand Versus Attribute Processing. Though analyzed for exploratory purposes, it was expected that RFS subjects would process by brand, at least initially, due to the need to determine attribute-values for the recommended brand as a tool for comparison to other available brands. Five of the 10 hybrid subjects processed by brand. An additional subject processed by attribute initially, but then acquired a recommendation and processed by brand for the remainder of the session. One hybrid subject acquired information too limited for classification. Contrary to expectations, only one of the three RFS subjects processed by brand. Using an alternative method of accomplishing the RFS strategy, the other two subjects developed lists of performance on selected attributes for particular brands, including the one recommended, and then made comparisons to the standard. Only three of the own-based subjects demonstrated clear processing by brand. Two others processed by brand during part of their session.

Selection of the Referrer/Information Source. Friend was the most commonly accessed source, with 75 percent of the own-based subjects and all but one of the hybrid subjects acquiring some of this attribute-value information. All hybrid subjects also acquired a recommendation from this source. Advertising and InStore Inspection were more popular than Consumer Reports or Salesperson/Clerk for own-based subjects. Hybrid subjects showed use of Consumer Reports about equal with that of In-Store and Salesperson/Clerk while Advertising received little use. These differences may be a function of the greater emphasis on hybrid decision making for subjects in the Stereo condition. For example, this higher-priced product may have made the acquisition of information from Consumer Reports more important despite the long time delay involved.

After obtaining a recommendation or key attributevalue information about one or more brands from a source, many subjects acquired attribute-value information about the same brand(s) from one or more additional sources. Three subjects indicated that this behavior was used to verify the knowledge of the original 
information source through comparison with information from other sources.

\section{DISCUSSION}

Results provide evidence for the existence of both the RFES and RFS hybrid brand choice strategies. RFES hybrid strategy may be particularly useful when the consumer feels the need to restrict the time spent in the decision process. RFS, while more time consuming than RFES, may offer the advantage of making the consumer an "instant expert." Future research should explore the personal and environmental factors that might account for differences in usage of these two major hybrid strategy rules. Possible additional hybrid strategies involving formation of the evaluative criteria, which could not be investigated given the design of the computer program used, should be explored.

The greater use of the RFES strategy for stereos meant that less information acquisition took place for stereos than for frozen pizza. Given the greater perceived risk involved in purchase for stereo receivers (higher price, greater complexity), the opposite finding would be expected. This may indicate that subjects used the RFES strategy to reduce perceived risk. However, since a number of other differences exist between the two product categories, further research is needed for clarification.

The apparent lack of use of the subcontracting strategy in its strict form was disappointing but not surprising. Despite use of an assistant to handle computer interaction, demand artifacts of the laboratory setting or subject curiosity with the system may have led subjects away from the very limited information acquisition involved in a subcontracted strategy.

\section{CONCLUSION}

The study results offer support for a broader view of consumer decision making as originally stated by Olshavsky and Granbois (1979) and Olshavsky and Rosen (1985) and provide additional empirical evidence as requested by Solomon (1986). This broader view needs to be further refined and delineated. The use of a referrer, not simply as an information source but as a participant in the decision itself, is an area deserving of further exploration.

[Received February 1986. Revised April 1987.]

\section{REFERENCES}

Bearden, William O. and Michael J. Etzel (1982), "Reference Group Influence on Product and Brand Purchase Decisions," Journal of Consumer Research, 9 (September), 183-194.

Bettman, James R. and Jacob Jacoby (1976), "Patterns of Processing in Consumer Information Acquisition," in Advances in Consumer Research, Vol. 3, ed. Beverlee B. Anderson, Ann Arbor, MI: Association for Consumer Research, 315-320.

Duncan, Calvin P. and Richard W. Olshavsky (1982), "External Search: The Role of Consumer Beliefs," Journal of Marketing Research, 19 (February), 32-43.

Hoyer, Wayne D. and Jacob Jacoby (1983), "Three-Dimensional Information Acquisition: An Application to Contraceptive Decision Making," in Advances in Consumer Research, Vol. 10, eds. Richard P. Bagozzi and Alice M. Tybout, Ann Arbor, MI: Association for Consumer Research, 618-623.

Olshavsky, Richard W. and Donald H. Granbois (1979), "Consumer Decision Making-Fact or Fiction." Journal of Consumer Research, 6 (September), 93-100.

- and Dennis L. Rosen (1985), "Use of Product-Testing Organizations' Recommendations as a Strategy for Choice Simplification," Journal of Consumer Affairs, 19 (Summer), 118-139.

Painton, Scott and James W. Gentry (1985), "Another Look at the Impact of Information Presentation Format," Journal of Consumer Research, 12 (September), 240244.

Park, C. Whan and V. Parker Lessig (1977), "Students and Housewives: Differences in Susceptibility to Reference Group Influence," Journal of Consumer Research, 4 (September), 102-110.

Rosen, Dennis L. and Richard W. Olshavsky (1987), “The Dual Role of Informational Social Influence: Implications for Marketing Management," Journal of Business Research, 15 (April), 123-144.

Russo, J. Edward and Larry D. Rosen (1975), "An Eye Fixation Analysis of Multialternative Choice," Memory and Cognition, 3 (May), 267-276.

Solomon, Michael R. (1986), "The Missing Link: Surrogate Consumers in the Marketing Chain," Journal of Marketing, 50 (October), 208-218. 